INTERNATIONAL JOURNAL
PHARMACEUTICAL SCIENCES
RESEARCH

Received on 09 September, 2012; received in revised form, 20 October, 2012; accepted, 26 December, 2012

\title{
DRUG USE EVALUATION OF SHENMAI INJECTION IN THE FIRST AFFILIATED HOSPITAL OF BENGBU MEDICAL COLLEGE: A PROSPECTIVE ANALYSIS
}

Qingping Shi ${ }^{11}{ }^{1}$, Feng Ding ${ }^{1}$, Ran Sang ${ }^{1}$, Yan Liu ${ }^{1}$ and Haoyu Yuan ${ }^{3}$

Department of Pharmacy, the first Affiliated Hospital of Bengbu Medical College ${ }^{1}$, Bengbu 233004, China College of Pharmacy, Bengbu Medical College ${ }^{2}$, Bengbu 233004, China

HeGongYe 416 Hospital $^{3}$, Chengdu 610051, China

\section{Keywords:}

Traditional Chinese medicine injection; Shenmai injection; Drug use evaluation; Drug use evaluation standard

Correspondence to Author:

Qingping Shi

Department of Pharmacy, the first Affiliated Hospital of Bengbu Medical College, Bengbu 233004, China

E-mail: sir_shi@126.com

\begin{tabular}{|c|c|}
\hline QUICK RESPONSE CODE & \multirow[b]{2}{*}{$\begin{array}{l}\text { IJPSR: } \\
\text { ICV- } 5.07\end{array}$} \\
\hline 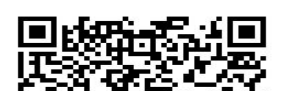 & \\
\hline 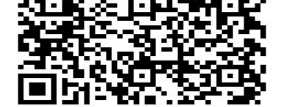 & $\begin{array}{c}\text { Website: } \\
\text { www.ijpsr.com }\end{array}$ \\
\hline
\end{tabular}

\begin{abstract}
Introduction: To investigate the evaluation pattern of Traditional Chinese medicine injection, establish the drug use evaluation(DUE) standards of shenmai injection, and implement of DUE programs of shenmai injection in the First Affiliated Hospital of Bengbu Medical College. Methods: Three hundred and thirty four patients were included in this retrospective analysis. The study duration was divided into two periods: cycle A: from November 2011 to December 2011; cycle B: from January 2012 to March 2012. A series of indices including indications, administration frequency, dosage, administration period, drug-drug interactions, adverse reactions and treatment efficiency were evaluated.
\end{abstract}

Results: The number of patients showed the indications of shenmai injection were 119 (77.27\%) and 168 (93.33\%) in cycle A and cycle B, respectively. The number of patients showed the contraindications of shenmai injection were $18(11.69 \%)$ and $12(6.67 \%)$ in cycle A and cycle B, respectively. Satisfactory outcomes were reported among all the patients with no serious adverse reactions reported.

Conclusions: The establishing of the standards of Traditional Chinese medicine injection should integrate the guidelines approved by drug use evaluation system. There are some shortcomings for the evaluation of Traditional Chinese medicine injections. The drug use evaluation of shenmai injection could promote the reasonable application of the injection in clinical practices.
INTRODUCTION: According to the Chinese State Food and Drug Administration, modern Chinese hospitals used as many as 147 kinds of herbal injections in China between 2004 and $2007^{1,2}$. Shenmai injection (SMI), a Chinese traditional prescription mainly consisted of two herbal components (Radix ginseng Rubra and Radix ophiopogonis) ${ }^{3}$, is widely used for the treatment of coronary atherosclerotic cardiopathy and viral myocarditis ${ }^{4,5}$. Although several trials have reported that shenmai injection might show a therapeutic effect on the cardiac function with pulmonary heart diseases 6,7 , the quality of these trials has not been evaluated systematically. Additionally, Traditional Chinese medicine (TCM) injection showed characteristics of large dosage, high concentration and long term administration, which may induce severe adverse 
reactions including changes of antibody titer, release of endogenous pyrogen and hemoclasis ${ }^{8}$. To regulate the administration of TCM injection in China, the Ministry of Health of the People's Republic of China established the Basic principles for clinical use of traditional Chinese medicine injections in December 2008. The guideline established the basic rules for the administration of TCM injection. However, no evaluation method for the clinical administration of TCM injection was mentioned.

According to our best knowledge, shenmai injection has been indicated for the treatment of shock as it could promote the blood circulation and enhance the microcirculation. Additionally, it has been frequently applied in the treatment of coronary heart disease ${ }^{9}$, angina pectoris ${ }^{10}$, myocardial ${ }^{11}$, viral myocarditis ${ }^{5}$, chronic cor pulmonale ${ }^{12}$ and heart failure ${ }^{13}$. For those with cancer, shenmai injection could be applied combined with chemotherapy and radiotherapy, which can protect the blood-producing function of the bone marrow and enhance the cellular immune function. However, some adverse reactions have been reported after shenmai injection including dry mouth, limb pain, drowsiness, allergic reaction, anorexia, erubescence, and even death ${ }^{14}$. We speculated that the occurrence of adverse reactions may be associated with the irregular administration pattern such as overdosage and off-label use caused by a lack of clinical guidance.

Drug use evaluation (DUE) is a system of ongoing, systematic, criteria-based evaluation of drug use that helps to ensure that medicines are used appropriately. As is known to all, DUE is drug specific and can be structured so that it will assess the actual process of prescribing, dispensing or administering a drug including the indications, dosage, and drug interactions.

Currently, both prospective analysis and retrospective analysis were used for the DUE. The prospective analysis mainly focused on the indications, drug dosage, dosage form, administration methods, treatment duration, treatment costs, contraindications, treatment efficiency, adverse reactions and drug interactions. The retrospective analysis mainly focused on the indications, most frequently used medicine, prescription, treatment costs, adverse reactions and drug interactions.
In this study, we aim to investigate the evaluation pattern of Traditional Chinese medicine injection, establish the drug use evaluation(DUE) standards of shenmai injection, and investigate the administration and prescription of shenmai injection in the First Affiliated Hospital of Bengbu Medical College according to drug use evaluation. This is the first report about the drug use evaluation of shenmai injection.

\section{METHODS:}

Study design: This prospective analysis was performed in the First Affiliated Hospital of Bengbu Medical College. The study duration was divided into two periods: cycle A: from November 2011 to December 2011; cycle B: from January 2012 to March 2012. The initial evaluation was performed in January 2012 to rectify and improve the administration pattern of the shenmai injection in various departments. The inclusion criteria included those received shenmai injection during the hospitalization. Moribund patients and patients with anticipated survival time of less than 1 month were excluded in this study. For those with repeated Shenmai injection, an interval of less than 15 days was considered as single administration, while an interval of more than 15 days was considered as the multiple administrations.

Drug use evaluation procedure: Drug use evaluation (DUE) was performed according to the guidelines approved by World Health Organization. In brief, DUE was consisted of eight steps:

1. To confirm the medicine and/or disease for evaluation.

2. To establish the evaluation criteria and indications.

3. To gain the approval of the medical authorities (such as the hospital and/or the Ministry of Health of the People's Republic of China).

4. To collect the data for drug utilization.

5. To rectify the drug administration pattern.

6. The second-round evaluation of the medicine.

7. To optimize the drug administration pattern and methods.

8. To rectify the evaluation criteria and summarize the clinical experiences. 
The detailed steps of the drug use evaluation of our study were summarized in Figure 1.

Evaluation criteria: According to our knowledge, the evaluation criteria for drug use included the information as follows:

1. Diagnosis standards.

2. Contraindication of the drug.

3. Drug-drug interaction standards.

4. Drug administration standards.

5. Treatment duration and drug dosage.

6. Drug dosage per day.

7. Appropriate or inappropriate standards.

Besides these standards, some other evaluation indices were needed to identify the accuracy of the collected data. There were two kinds of indices including absolute indices (such as the judgment on the drug indications) and the indices evaluated by deviation and percentage (beyond the deviation was considered as non-standard).
Data collection and Analysis: Data collection format was developed according to WHO recommendation and the DUE standards. Each patient chart was examined looking at key in formations like indications, dose, dose frequency, dose duration, the potential for drug -drug interaction, adverse reactions and treatment efficiency. Student $t$ test was applied to compare the data obtained from cycle A and cycle B. $\mathrm{P}<0.05$ demonstrated significant difference.

RESULTS: In this study, a drafted DUE was established according to the DUE guidelines approved by American Society of Health-System Pharmacists (ASHP) and the Basic Principles for Clinical Use of Traditional Chinese Medicine Injections approved by Ministry of Health of the People's Republic of China. To confirm the feasibility and accuracy of this draft, we consulted with some senior experts for the revision of this draft. Additionally, a two-week drug use evaluation was performed. Finally, we collected the information and made the final draft according to the DUE in this prospective study. The DUE criteria for Shenmai injection is shown in Table 1.

\section{TABLE 1: DUE CRITERIA FOR SHENMAI INJECTION}

\begin{tabular}{|c|c|c|}
\hline Indicator & Criteria & Threshold (\%) \\
\hline Indication & $\begin{array}{c}\text { Coronary heart disease, Viral myocarditis, Chronic cardiopulmonary disease, Granulocytopenia, } \\
\text { Adjuvant chemotherapy, Type of deficiency of both Qi and Yin }\end{array}$ & 95 \\
\hline Contraindications & $\begin{array}{c}\text { Those with history of drug allergy and/or family history of drug allergy, Neonates and babies, } \\
\text { Pregnant women }\end{array}$ & 90 \\
\hline Dosage & $\begin{array}{l}\text { 2 } \sim 4 \mathrm{ml} \text { via intramuscular injection. Once per day. } \\
\text { General treating: Intravenous drip, } 10 \sim 60 \mathrm{ml} \text { per day. } \\
\text { Shock: The dosage should be more than } 200 \mathrm{ml} \text {. } \\
\text { Cardiovascular disease: Add 10-40ml shenmai injection into the glucose injection. } \\
\text { Cancer: Add } 40-60 \mathrm{ml} \text { shenmai injection into the glucose injection. }\end{array}$ & 85 \\
\hline Direction of usage: & $\begin{array}{c}\text { Intravenous push was not allowed. } \\
\text { The velocity of iv gtt should be }<40 / \mathrm{min} \\
\text { Glucose }(5 \%) \text { injection is preferred for the solvent of the Shenmai injection. }\end{array}$ & 85 \\
\hline Duration & For those with carcinoma and/or CVD: 10-15 days. Single injection per day. & 95 \\
\hline Drug interactions & $\begin{array}{l}\text { Could not administrated together with the following drugs: } \\
\text { Black falsehellebore root, Faeces Trogopteri, glyceroal and fructose, antibiotics, penicillins. } \\
\text { Additionally, the Maishen injection could not be administrated via iv gtt with the other drugs. }\end{array}$ & 95 \\
\hline $\begin{array}{l}\text { Adverse drug } \\
\text { reaction }\end{array}$ & $\begin{array}{l}\text { Itch of skin, rash, skin redness, cyanosis, urticaria, flushed face, drug fever, anaphylactic shock, } \\
\text { phlebitis, dyspnea, tachypnea, breathlessness, obstruction of respiratory tract, upper respiratory } \\
\text { tract infection, tachycardia, angina pectoris, heart failure, palpitation, nausea, vomiting, upper } \\
\text { gastrointestinal hemorrhage, singultus, unconsciousness, dysphoria, mental stress, coma, } \\
\text { headache, chest pain, back pain, stomachache, general malaise, numbness, liver function injury } \\
\text { and scanty dark urine. }\end{array}$ & $>0.4 \sim 0.5 \%$ \\
\hline Outcome & Most of the patients showed satisfactory outcomes. No adverse reactions were reported. & $90 \%$ \\
\hline
\end{tabular}


The number of patients included in Cycle A and B were 154 and 180, respectively. The averaged ages for cycle $A$ and $B$ were $41.9 \pm 16.1$ yrs and $40.6 \pm 14.2 \mathrm{yrs}$, respectively. Most of the patients were female (cycle A: $63.64 \%$, cycle B: $66.11 \%$ ). No significant difference was noted between the clinical data before the study.

The number of patients showed the indications of Shenmai injection were $119(77.27 \%)$ and 168 (93.33\%), respectively (Table 2 ). The number of patients showed the contraindications (mainly due to history of allergy) were 18 and 12 in cycle A and B, respectively. Compared with Cycle A, significant improvement was noted in the patients with indications and contraindications of Shenmai injection in Cycle $B(P<0.01)$.

The averaged dosage of Shenmai injection in Cycle $A$ and $B$ were $64.78 \pm 15.86 \mathrm{~mL}$ and $69.61 \pm 12.77 \mathrm{~mL}$, respectively (Table 3 ). Statistical difference was noted TABLE 2 INDICATIONS FOR THE SHENMAI INJECTION DURING DUE

\begin{tabular}{ccc}
\hline Indications & \multicolumn{2}{c}{ Case number } \\
\cline { 2 - 3 } & Cycle A & Cycle B \\
\hline Adjuvant chemotherapy & $63(40.91 \%)$ & $80(44.44 \%)$ \\
Granulocytopenia & $23(14.94 \%)$ & $28(15.56 \%)$ \\
Cytopenia & $8(5.19 \%)$ & $6(3.33 \%)$ \\
Bone marrow hyperplasia & $5(3.25 \%)$ & $2(1.11 \%)$ \\
Coronary heart disease & $15(9.74 \%)$ & $19(10.56 \%)$ \\
Viral myocarditis & $5(3.25 \%)$ & $18(10.00 \%)$ \\
Chronic cardiopulmonary disease & $5(3.25 \%)$ & $17(9.44 \%)$ \\
Pelvic lump & $10(6.49 \%)$ & $2(1.11 \%)$ \\
Hyperthyroidism & $5(3.25 \%)$ & $1(0.56 \%)$ \\
Diabetes & $4(2.60 \%)$ & $1(0.56 \%)$ \\
Rheumatoid arthritis & $4(2.60 \%)$ & $3(1.1 \%)$ \\
Brain infarction & $3(1.95 \%)$ & $1(0.56 \%)$ \\
Cholecystitis & $2(1.30 \%)$ & $1(0.56 \%)$ \\
Hypertension & $2(1.30 \%)$ & $1(0.56 \%)$ \\
\hline
\end{tabular}

TABLE 3 DRUG USE EVALUATION OF SHENMAI INJECTION OF CYCLE A AND CYCLE B

\begin{tabular}{ccc}
\hline Items & $\begin{array}{c}\text { Number of patients consistent with the standards in } \\
\text { cycle A }\end{array}$ & $\begin{array}{c}\text { Number of patients consistent with the standards in } \\
\text { cycle B }\end{array}$ \\
\hline Indication & $119(77.27 \%)$ & $168(93.33 \%)$ \\
Contraindications & $136(88.31 \%)$ & $168(93.33 \%)$ \\
Dosage & $104(67.53 \%)$ & $103(57.22 \%)$ \\
Direction of usage & $5(3.25 \%)$ & $8(4.44 \%)$ \\
Duration & $150(97.40 \%)$ & $175(97.22 \%)$ \\
Drug interactions & $103(66.88 \%)$ & $130(72.22 \%)$ \\
Adverse reactions & $0(0)$ & $1(0.56 \%)$ \\
Outcome & $154(100 \%)$ & $180(100 \%)$ \\
\hline
\end{tabular}

in the averaged dosage in Cycle $A$ and $B(P<0.05)$. The number of patients with overdosages of Shenmai injection were 50 (32.47\%) and 77 (42.78\%) in Cycle A and $B$, respectively. No significant difference was noted in the number of patients with overdosages of Shenmai injection $(P>0.05)$. With regards to the treatment duration, only part of the patients received Shenmai injection for more than 15 days. For the administration method of the injection, iv gtt was used in both cycles. The velocity of the administration was kept at less than 40 drops per minute. Number of patients consistent with the standardized direction of usage in cycle $A$ and B were $5(3.25 \%)$ and $8(4.44 \%)$, respectively.

For the adverse reactions, only 1 patient showed slight rash and itch of skin. Additionally, the number of patients with potential drug interaction with glycerol and fructose, antibiotics as well as penicillins, were 51 (33.12\%) and $50(27.78 \%)$, respectively. 
DISCUSSION: The priority of DUE was to establish the standards for drug use evaluation, which could provide guidelines and references for the drug usage ${ }^{15}$. The drug use evaluation standards of the shenmai injection integrated the responsibilities of the doctors, nurses and pharmaceutists. The doctors should select the correct indications and exclude the contraindications. The pharmaceutists should monitor the medication and the adverse reactions before the injection. The nurses should master the velocity of the transfusion. Our study indicated the administration of shenmai injection in our hospital was in a reasonable stage. However, some further studies were needed to improve the clinical outcomes.

In this study, the clinical application of shenmai injection in our hospital was evaluated. Satisfactory outcomes were obtained in both cycles, among which patients of cycle B showed significant improvement in the identification of the indications and contraindications compared with cycle A. However, a large number of patients were observed with overdosage and inappropriate administration of shenmai injection.

According to the previous reports, the administration pattern and dosage might be associated with allergy ${ }^{16}$, 17. Therefore, it is necessary to check the history of allergy before the administration of the shenmai injection.

The final goal for DUE was to improve the drug administration process, based on which to improve the outcome of the patients. Therefore, DUE has been considered to be closely related with the quality control of the medical department. In our study, a series of indices including indications, contraindications, application frequently, drug dosage, treatment duration, drug interactions, adverse reactions, treatment efficiency were investigated to establish a standard DUE for the shenmai injection.

The study duration was divided into two periods: cycle A: from November 2011 to December 2011; cycle B: from January 2012 to March 2012. Compared with cycle A, significant improvement was noted in the patients with indications and contraindications of Shenmai injection in Cycle B $(P<0.01)$.
There has been a litany of poor outcomes in recent years following administration of Chinese herbal injection. As a lack of randomly controlled trials, the treatment efficiency of some herbal injection was not appropriately evaluated including shenmai injection. Based on the administration of shenmai injection in our hospital, we conducted this prospective study to analyze the administration of shenmai injection. We hope our study can provide some basic information for the clinical physicians.

Our study showed the following shortcomings: For the treatment efficiency, most of the patients received drug combination therapy. Additionally, shenmai injection was mainly used as the adjuvant drug for chemotherapy. Therefore, it was difficult to evaluate the efficiency of shenmai injection.

CONCLUSIONS: The present study showed the clinical application of Shenmai injection in the First Affiliated Hospital of Bengbu Medical College. We hope to promote the appropriate administration of shenmai injection through a prospective analysis. In general, the establishing of the standards of shenmai injection should integrate the guidelines approved by drug use evaluation system, which could promote the reasonable application of the injection in clinical practices. In addition, the safety of shenmai injection would be improved if controlled clinical trials as well as studies examining the toxicology and allergenic potential were conducted. We especially underscore careful application of shenmai injection in clinics to avoid any misuse and/or adverse reactions.

ACKNOWLEDGEMENTS: We thank the anonymous reviewers for their reviewing of this manuscript.

COMPETING INTERESTS: The authors declare that they have no competing interests.

AUTHORS' CONTRIBUTIONS: QPS: Study design and conception, data/statistical analyses, drafting the manuscript; FD: Study design, data/statistical analyses, Critical revision of the article; RS, YL and HYY: data collection and statistical analyses. All authors contributed to writing of the final manuscript; All authors read and approved the final manuscript. 


\section{REFERENCES:}

1. Ji K, Chen J, Li M, Liu Z, Xia L, Wang C, et al. Comments on serious anaphylaxis caused by nine Chinese herbal injections used to treat common colds and upper respiratory tract infections. Regul Toxicol Pharmacol. 2009, 55, 134-138.

2. Yang $\mathrm{M}$, Zhang $\mathrm{XX}$, Wan $\mathrm{N}$. Comments on the temporary prohibition of Yuxingcao (Houttuynia cordata) herbal injection use in China. Journal of Jianxi University of TCM. 2007, 19, 8183.

3. Wang L, Wang W, Zhao X, Jin L, Bai $Y$, Yao H, et al. Effect of Shenmai injection, a traditional Chinese medicine, on pulmonary dysfunction after tourniquet-induced limb ischemiareperfusion. J Trauma. 2011, 71, 893-897.

4. Xia CH, Sun JG, Wang GJ, Shang LL, Zhang XX, Zhang R, et al. Herb-drug interactions: in vivo and in vitro effect of Shenmai injection, a herbal preparation, on the metabolic activities of hepatic cytochrome P450 3A1/2, 2C6, 1A2, and $2 \mathrm{E} 1$ in rats. Planta Med. 2010, 76, 245-250.

5. Grohe C, Kahlert S, Lobbert K, Neyses L, van Eickels M, Stimpel $M$, et al. Angiotensin converting enzyme inhibition modulates cardiac fibroblast growth. J Hypertens. 1998,16, 377-384.

6. Chen $\mathrm{H}$, Jia L. Effects of shenmai injection on cardiac function and hemorheology in patients with acute exacerbation of senile chronic pulmonary heart disease. Chin J Cardiovasc Rehabil Med. 2003, 12, 274-276.

7. Du Y, Jia L. Therapeutic effect of shenmai injection for ad. J Zhangjiakou Med Coll. 1998, 15, 16-17.

8. Ning GL, Sun LQ. The status quo of Traditional Chinese injection Practical Journal of Cardiac cerebral pneumal and vascular disease. 2011, 19, 239-242.
9. Su X, Ma Y, Huang R, Wang X, Wang Y. Effects of shenmai injection on blood SOD activity and MDA level in senile patients with coronary heart disease. J Tradit Chin Med. 2005, 25, 50-53.

10. Wu YG, Zhao S, Fan XJ, Zhao WQ. Guanxin Ning injection combined with shenmai Injection for ustable angina pectoris. Journal of Anhui Traditional Chinese Medical College. 2011, 30, 30-32.

11. Guo SP, Zhang YZ. [Study on effect of shenmai injection in protecting myocardium against ischemia-reperfusion injury in thrombolytic therapy with urokinase for acute myocardial infarction patients evaluated by $99 \mathrm{mTc}-\mathrm{MIBI}$ myocardial imaging]. Zhongguo Zhong Xi Yi Jie He Za Zhi. 2001, 21, 108-110.

12. Wang $H$. Therapeutic effect of Shenmai injection on patients with chronic cor pulmonale. China Tropical Medicine. 2011, 11, 1-5.

13. Liu Z, Cheng $X$, Zhang C. [Observation on effect of shenmai injection in treating patients of congestive heart failure]. Zhongguo Zhong Xi Yi Jie He Za Zhi.1998, 18, 212-213.

14. Li JS, Wang HF, Li SY, Yu XQ, Wang ZW. Shenmai injection for chronic pulmonary heart disease: a systematic review and meta-analysis. J Altern Complement Med. 2011, 17, 579-587.

15. Al-Mendalawi MD. Drug use evaluation of antibiotics prescribed in a Jordanian Hospital outpatient and emergency clinics using WHO prescribing indicators. Saudi Med J. 2008, 29, 1362; author reply 1362.

16. Wang L, Yin T, Zhu MW. Retrospective study of adverse drug reactions of Shenmai injection. Central South Pharmacy. 2007, 5, 275-279.

17. Wang $X M, Y u Y X$, Qian F. Analysis of transfusion vector for shenmai injection West China Journal of Pharmaceutical Sciences. 2010, 25, 249-250.

How to cite this article:

Shi Q, Ding F, Sang R, Liu Y and Yuan H: Drug use evaluation of Shenmai injection in the first affiliated hospital of Bengbu Medical College: A Prospective Analysis. Int J Pharm Sci Res. 2013; 4(1); 225-230. 